\title{
Diseño de un plan de visibilidad científica e identidad digital para los investigadores de la Universidad de Guadalajara (México)
}

Design of a plan for the scientific visibility and digital identity of the researchers of the University of Guadalajara (México)

\author{
Martin-Adalberto TenA-EsPinOZA-DE-Los-MonTERos (1), Alicia GARcíA-Holgado (2), José-Antonio \\ Merlo-Vega (3), Francisco J. García-Peñalvo (4)
}

(1) Coordinación de Bibliotecas - Red de Bibliotecas (REBIUdeG), Universidad de Guadalajara, México, mtenaespinoza@udgvirtual.udg.mx; $(2,4)$ Grupo de Investigación en Interacción y eLearning, Universidad de Salamanca, España, \{aliciagh | fgarcia\} @usal.es; (3) Departamento de Biblioteconomía y Documentación, Universidad de Salamanca, España, merlo@usal.e

\section{Resumen}

Se documenta, sistematiza y valora la prueba piloto para la gestión y desarrollo de la visibilidad científica y la identidad digital de los investigadores realizada en el Centro de Investigaciones en Comportamiento Alimentario y Nutrición de la Universidad de Guadalajara, Méjico. Se exponen las intenciones, la justificación y el alcance de dicha experiencia, se describen el diseño, los contenidos y las etapas de desarrollo, se presentan los resultados obtenidos y se reflexiona sobre un posible futuro diseño de una estrategia institucional para la promoción, comunicación y posicionamiento académico en la web 2.0 de la comunidad de institutos y centros de investigación de la UdeG.
\end{abstract}

Palabras clave: Visibilidad científica. Identidad digital. Producción científica. Comunicación científica. Ciencia 2.0. Ecosistema tecnológico. Centros de investigación. Universidad de Guadalajara. Méjico.

\section{Introducción}

En el contexto de una sociedad red, de la sociabilidad de Internet y de la evolución de la Web hacia la Web 2.0 (Castells, 2006; O’Reilly 2005), la gestión de una identidad digital se ha convertido en un proceso que está complementando y acompañando el desarrollo de la identidad individual y la identidad humana (ver Fundación Telefónica, 2013, p.3).

Si partimos de la definición más básica del concepto de identidad, entiendo a ésta como el "conjunto de rasgos propios de un individuo o de una colectividad que los caracteriza frente a los demás" (RAE, 2104), el concepto de identidad digital puede ser definido a partir de los "los rasgos del individuo que encontramos digitalizados y que están a disposición de los demás" (Pérez, 2012), a partir de "plataformas digitales que representan el vehículo que enmarca la identidad digital" (Fernández, 2012).

\begin{abstract}
The experience gained in a pilot test for the management and development of the scientific visibility and digital identity of the researchers of the Universidad de Guadalajara's Research Center on Feeding Behavior and Nutrition is documented, systematized and assessed. The intentions, justification and reach of this experience are described in this paper, as well as its design, contents and development stages. The results obtained with the implementation of the pilot test are shown. A reflection on a possible future design of an institutional strategy for the promotion, communication and academic positioning in the 2.0 web of the community of research centers and institutes of the UdeG is made.
\end{abstract}

Keywords: Scientific visibility. Digital identity. Scientific production. Scientific communication. Science 2.0. Technological ecosystem. Research centers. Universidad de Guadalajara. Mexico.

De esta manera, la irrupción y el establecimiento de entornos cada vez más digitales en nuestras vidas, y muy particularmente en los mecanismos, medios y ambientes en los cuales habíamos venido desarrollando y sustentando nuestros procesos de comunicación, están conformando nuevos escenarios y nuevas formas de relacionarnos y comunicarnos: nuevas formas de identificarnos.

Estos inéditos entornos y contextos digitales, demandan el desarrollo de nuevas competencias y habilidades sociales, culturales, académicas y productivas. Estas exigencias se configuran tanto de forma individual como colectiva.

En el ámbito de la ciencia y el desarrollo científico, la identidad digital se configura como una nueva dimensión de la calidad de la investigación (Fernández y González, 2015), así como en un mecanismo de participación y crecimiento académico y científico, donde identidad y repu- 
tación se configuran en un plano digital y en un marco donde las infraestructuras digitales (entornos digitales) han promovido según Cobo y Naval (2013, p. 53):

[...] new facets of knowledge generation (wikis, escience, online education, distributed R\&D, open innovation, open science, peer-based production, online encyclopedias, user generated content) and new models of knowledge circulation and distribution (e-journals, open repositories, open licenses, academic podcasting initiative, etc.).

La sucesión de los antiguos "entornos análogos" por "entornos altamente digitales" ha redefinido los modelos de producción y comunicación científica, impactando y afectando a sus principales actores: académicos e investigadores (Esposito, 2016). Así pues, nos encontramos ante la necesidad de que académicos e investigadores conozcan y utilicen herramientas y estrategias que les permitan posicionarse y ser reconocidos en los actuales entornos digitales en los que la academia y la ciencia actualmente se desarrollan y evolucionan: la Ciencia 2.0 en el marco de una Web 2.0 (European Commission, 2014; Rathi y Given, 2010; Science Europe 2014, Zapata, 2011).

Con el presente trabajo pretendemos indagar a partir de un estudio de caso, el estado que guarda la gestión y desarrollo de identidades digitales académicas en un grupo de investigadores concreto, y proponer un plan de implementación para ello.

\section{Objetivo}

El objetivo del presente trabajo es, en una primera etapa, sistematizar y documentar en una primera etapa, la implementación de una experiencia piloto sobre la gestión y desarrollo de la visibilidad científica y la identidad digital de los investigadores en un centro de investigación de la UdeG. Nuestro propósito es identificar y determinar las condiciones y los cambios que se requieren para generar una estrategia a nivel institucional de promoción, comunicación y posicionamiento académico en la Web 2.0 para la comunidad de institutos y centros de investigación de la UdeG. La sistematización y documentación de la implementación, desarrollo y resultados de la prueba piloto nos permitirá entre otras cosas: observar y monitorear las reacciones de los participantes implicados; cuantificar y analizar resultados e impactos de la implementación, e identificar oportunidades de mejora para futuras implementaciones y pruebas pilotos.

En una segunda etapa, los resultados e insumos obtenidos se emplearán para la elaboración de una hoja de ruta y una guía de mejores

prácticas que sirvan de directrices y soporte para la generación de una estrategia a nivel institucional.

\section{Estudio de caso}

\subsection{La Universidad de Guadalajara}

La Universidad de Guadalajara (UdeG) es la segunda institución pública de educación superior de México en historia y tamaño, con 200 años de historia en el occidente del país, ha sido protagonista del desarrollo educativo, científico, tecnológico y cultural que sustenta el desarrollo del Estado de Jalisco, la Región Occidente del país y de México en su conjunto.

La UdeG conforma la Red Universitaria del Estado de Jalisco. Con sus seis centros universitarios temáticos, nueve centros universitarios regionales, un Sistema de Universidad Virtual y un Sistema de Educación Media Superior, tiene presencia en todas sus regiones (Figura 1).
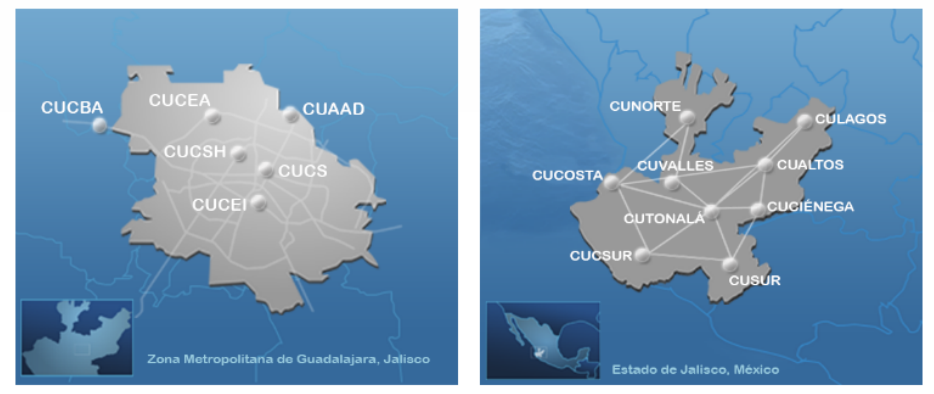

\section{Centros Universitarios Temáticos - CUAAD - Arte, Arquitectura y Diseñoø \\ CUCBA - Ciencias Biologicas y Agropecuarias \\ CUCEA-Ciencias Económico-Administrativas \\ CUCEl-Ciencias Exactas e Ingenierías 0 \\ cucs - Ciencias de la Salud $\bullet$ \\ CUCSH - Ciencias Sociales y Humanidades}

Sistema de Educación Media Superior。

Figura 1. Red Universitaria del Estado de Jalisco

Durante el ciclo escolar 2015-2016 la comunidad universitaria estuvo integrada por $291 \mathrm{mil}$ 67 universitarios, de los cuales 265 mil 167 eran estudiantes (146 mil 502 en nivel medio superior y 118 mil 665 en el nivel superior), 9 mil 588 trabajadores administrativos y 16 mil 312 académicos (UdeG, 2015, p. 5). La oferta educativa de la UdeG la conforman 30 programas de nivel medio superior, 143 pro-gramas de pregrado, 210 programas de pos-grado y 12 programas de otros niveles (COPLADI, 2016). 
3.2. Visibilidad e impacto de la producción científica de la Universidad de Guadalajara

La comunidad académica de la UdeG se distingue por ser una de las más importantes de México. En el rubro de la producción científica, de enero del 2010 a noviembre de 2015, la UdeG había producido 2.636 artículos en revisas indexadas en Scopus y 2.710 en revistas indexadas en Web of Science (UdeG, 2015, p. 27). Esta producción la sitúa como la sexta institución de educación superior pública de México en cuanto a publicación de artículos científicos reconocidos por estos índices a lo largo de los últimos 5 años (DGEI, 2015).

A nivel de Rankings Internacionales, la UdeG tiene presencia solamente en tres de los principales rankings globales: SIR IBER y SIR de SCImago Research Group, Webometrics del Laboratorio de Cibermetría del CSIC, y en el QS y QS Latin America de Quacquarelli Symonds (DGEI, 2015).

En la edición 2015 del SCImago Institutions Rankings Iberoamérica (SIR IBER) correspondiente al quinquenio 2009-2013, la UdeG ocupó las siguientes posiciones: posición 91 en el ranking lberoamericano (IBE), posición 47 en el ranking Latinoamericano y del Caribe (LAC) y la posición 5 en el ranking nacional (CO), manteniendo sus mismas posiciones con respecto a la edición 2013 en los rankings IBE y CO y subiendo tres posiciones en el ranking LAC (SClmago, 2013, 2015). En lo relativo a la edición global del SCImago Institutions Rankings (SIR), la UdeG ocupó la posición 1770 en el año 2014 y la posición 1889 en el año 2013 (SCImago, 2016). En lo que respecta al QS en su edición global (QS World University Ranking) la UdeG ocupó en el año 2015/16 el rango 651-700, y en el ámbito Latinoamericano (QS Latin America) la posición 42 (QS Quacquarelli Symonds, 2016). En lo que respecta al Ranking Web de Universidades (Edición 2016.1.1) la UdeG ocupa las siguientes posiciones: 784 del Ranking Mundial, 270 del Ranking Continental, 31 del Ranking Latinoamericano y 6 del Ranking por País (Laboratorio de Cibermetría, 2016).

\subsection{La visibilidad digital de los investigadores} de la Universidad de Guadalajara

A finales del año 2015, el Laboratorio de Cibermetría del CCHS-CSIC, elaboró un ranking global experimental para universidades basado en los perfiles institucionales de los investigadores hasta hoy generados en la plataforma de Google Scholar y su aplicación Google Scholar Citations. La posición que ocupa la UdeG en dicho ranking es la 1585 de un total de 2106 universi- dades (Laboratorio de Cibermetría, 2015a); las universidades mexicanas mejor posicionadas ocupan los lugares 363 (Centro de Investigación y de Estudios Avanzados del Instituto Politécnico Nacional - CINVESTAV/IPN) y 364 (Universidad Nacional Autónoma de México - UNAM). A partir de esta iniciativa, se han elaborado versiones de rankings para científicos segmentándolos por países. Dentro del ranking de científicos de instituciones mexicanas de acuerdo a sus perfiles públicos en Google Scholar Citations, las posiciones 138, 267, 281, 470 y 643, son las primeras cinco posiciones que la UdeG ocupa de un total de 122 menciones en un ranking que incluye 3800 perfiles mexicanos (Laboratorio de Cibermetría, 2015b).

Otros datos relativos a la visibilidad digital y la construcción de identidades digitales en los investigadores de la UdeG nos los brinda la red social ResearchGate. Dicha red social académica reportaba en octubre de 2015 la creación de 8 millones perfiles de usuarios (ResearchGate, 2015). A este universo de perfiles digitales, la UdeG aporta la cantidad de 1,811 perfiles, los cuales en función del número de artículos que han aportado (1,871 artículos) y el impacto que han tenido dentro de la comunidad de usuarios de ResearchGate (RGScore), posicionan a la UdeG como la quinta universidad mexicana en el RGScore para México. Es conveniente resaltar la gran disparidad que existe entre el número de perfiles creados, el número de artículos aportados y el RGScore de la primera universidad mexicana, la UNAM, con el resto de las universidades mexicanas (Figura 2) (ResearchGate, 2016).

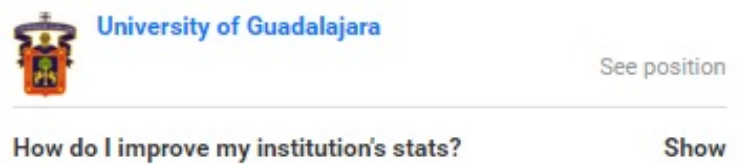

TOP 5 BY TOTAL RG SCORE IN MEXICO -

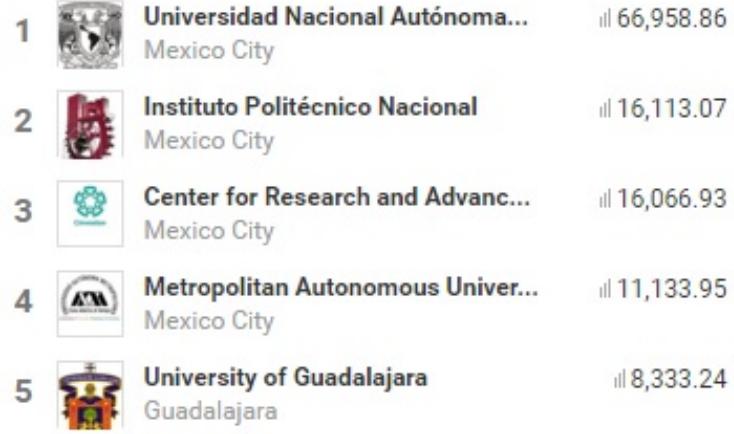

Figura 2. Posición de la UdeG ante universidades mexicanas en el RGScore 
Los datos de visibilidad, impacto y numero de perfiles registrados en estas dos plataformas sociales académicas, sumado a una limitada y pobre presencia de la UdeG en los distintos rankings internacionales de universidades, nos permiten inferir que existe una escasa y deficiente gestión de identidades digitales entre las comunidades docentes y científicas de la UdeG, así como también de las identidades digitales institucionales de los centros e institutos de investigación de esta universidad.

\section{Metodología}

La prueba piloto se configuró a partir de tres acciones dirigidas a un grupo de investigadores adscritos a uno de los centros de investigación de la UdeG: 1) un programa de cursos de formación docente; 2) una estrategia para la visibilidad científica y la identidad digital; y 3) la creación de un entorno tecnológico para la gestión del conocimiento.

Estas acciones se configuraron y desarrollaron en tres etapas: 1) etapa de diagnóstico y diseño, 2) etapa de desarrollo, implementación y monitoreo y 3) etapa de evaluación y mejoras. Los pasos y las acciones realizadas en cada una de las etapas se resumen en las siguientes fases:

\subsection{Etapa de diagnóstico y diseño}

\subsubsection{Selección del instituto o centro de investi- gación}

La UdeG cuenta con 121 institutos o centros de investigación (COPLADI, 2016), los cuales se encuentran distribuidos a lo largo de la Red Universitaria. Estos centros se especializan y atienden distintas áreas y ramas del conocimiento y la ciencia, y forman parte de la infraestructura científica y académica de la universidad.

La selección del instituto o centro de investigación para esta prueba se hizo en función de indicadores de calidad y productividad científica y académica (publicaciones científicas, proyectos en marcha, programas de posgrado, actividades de docencia, obtención de reconocimientos, generación de convenios y acuerdos de cooperación), así como también en función de facilidades técnicas y administrativas que permitieran llevar a cabo la prueba piloto.

El centro de investigación seleccionado fue el Centro de Investigaciones en Comportamiento Alimentario y Nutrición (CICAN), el cual está adscrito al Centro Universitario del Sur (CUSur). El CICAN tiene como propósito promover y efectuar investigación multidisciplinaria del comportamiento alimentario, relacionando la investigación básica en psicología con la investigación desarrollada en antropología, nutrición, genética, fisiología, bioquímica e inmunología.

\subsubsection{Recopilación y análisis de información}

Se solicitó y se contó con información relativa a los perfiles del personal docente e investigador del CICAN, así como de las actividades académicas y de investigación que como institución ha venido desarrollando el centro de investigaciones.

\subsubsection{Diseño y aplicación del instrumento de evaluación diagnostica}

El instrumento de evaluación diagnostica se elaboró con base en la revisión de literatura especializada, principalmente aquella que reportaba la realización de otros estudios acerca del uso de redes sociales y redes sociales académicas por parte de investigadores (Campos, Rúas, 2016; Clavel, Maruenda, 2010; Gil, 2015a, 2015b; Gómez, Roses, Farias, 2012; González, Iglesias, Codina, 2015; Rifón, Goicochea, Fenollera, 2016; Uribe, 2015).

El instrumento de evaluación diagnostica consistió en una encuesta de 25 preguntas agrupadas en 4 secciones: a) datos generales, b) conocimiento y uso de redes sociales, c) participación en redes sociales académicas y d) percepción de las redes sociales académicas.

La encuesta fue diseñada y distribuida a través de la aplicación Formularios de Google, y se hizo llegar a la totalidad del personal docente e investigador del CICAN (13 funcionarios). También fue incluido un grupo de estudiantes ( 5 participantes) que fungen como asistentes y que cursan estudios de posgrado en el programa de Doctorado en Ciencias del Comportamiento con orientación en Nutrición y Alimentación, que el CICAN gestiona. La encuesta también se hizo llegar a un grupo de 10 profesores-investigadores invitados adscritos a otros centros de investigación del CUSur, pero que colaboran activamente con el CICAN. Todos los participantes recibieron en sus cuentas de correo electrónico un enlace directo y restringido a la encuesta.

La encuesta de evaluación diagnóstica fue realizada con carácter obligatorio entre el 01 y 08 de mayo 2016. Se obtuvo la respuesta de 27 de los 28 participantes convocados, lo que representa un $96 \%$ de los encuestados.

Una primera evaluación de los resultados se realizó entre los días 09 y 21 de mayo con la intención de identificar las limitaciones y deficiencias en el conocimiento y uso de redes sociales académicas de los participantes. Estos 
resultados también fueron tomados en cuenta para el diseño de los contenidos de los cursos de formación docente y para la estrategia a implementar. Un segundo análisis y una reflexión sobre las respuestas de la encuesta se presentan en la sección de resultados de este trabajo, así como también en las conclusiones.

\subsubsection{Diseño del programa de cursos de forma- ción docente}

El programa de cursos de formación docente se diseñó a partir la consulta documental de cursos, talleres y experiencias existentes en el tema (Álvarez de Toledo, 2013; Alonso, 2016; Barrionuevo, 2015; Borras, 2016; Moya, 2015; Orduña, López, 2014; 2016; Penkova, 2016; REBIUN, 2011). También fueron consideradas las oportunidades detectadas a través del análisis de la información arrojada en la encuesta de evaluación diagnostica.

\subsubsection{Diseño de la estrategia}

El diseño de la estrategia para la visibilidad científica y la identidad digital se configuró con base en tres líneas de acción: 1) creación y desarrollo de perfiles en redes sociales, redes sociales académicas y recursos de la Web 2.0 para cada profesor-investigador del CICAN; 2) creación y desarrollo de perfiles institucionales en redes sociales y recursos de la Web 2.0 para el CICAN; y 3) configuración del ecosistema tecnológico del CICAN.

\subsubsection{Diseño del entorno tecnológico para la gestión del conocimiento}

El ecosistema tecnológico del CICAN se basa en una experiencia tecnológica previa llevada a cabo en el programa de doctorado Formación en la Sociedad del Conocimiento de la Universidad de Salamanca (García-Holgado, GarcíaPeñalvo, Rodríguez, 2015). El ecosistema está formado por un conjunto de cuatro componentes desarrollados ad-hoc (plataformas), con un alto grado de integración entre sí que tienen como objetivo impulsar la gestión del conocimiento generado dentro del CICAN.

\subsection{Etapa de desarrollo, implementación y monitoreo}

El desarrollo y la implementación piloto de este plan de gestión se llevó a cabo durante el mes de junio de 2016 en el CICAN.

El monitoreo se ha venido realizando desde la finalización de la prueba piloto y se mantiene vigente con dos intenciones: 1) dar soporte a los profesores-investigadores, así como al propio
CICAN como organización, y 2) recopilar información y experiencias posteriores a la implementación de la prueba piloto, así como de la evolución y trascendencia de los impactos y resultados de la misma.

\subsection{Etapa de evaluación y mejoras}

\subsubsection{Documentación de la experiencia}

La documentación será realizada en dos fases, la primera fase ha sido cubierta con la elaboración de este trabajo al documentar la experiencia de implementación; la segunda fase cubrirá y reportará avances e impactos futuros derivados de la implementación de la prueba piloto.

\subsubsection{Evaluación y correcciones}

La evaluación final de la implementación del plan y la prueba piloto consta de dos momentos. Un primer momento es el presente trabajo, el cual documenta los resultados y reflexiones de la primera etapa del proceso de evaluación y mejora. Esta primera etapa se enfocó en evaluar los impactos más inmediatos a partir de la implementación de la prueba piloto: la creación y gestión de las identidades de los profesoresinvestigadores y, del propio CICAN; así como su adaptación y relación con el entorno tecno-lógico configurado. En esta primera evaluación se aplicaron dos encuestas, la primera de ellas estuvo compuesta por 5 preguntas relativas a la experiencia en la creación de perfiles académicos por parte de los participantes; mientras que la segunda de las encuestas contó con 8 preguntas sobre la satisfacción con los contenidos y las actividades de los cursos de formación docente implementados. Con el análisis de estos resultados se buscó detectar oportunidades de mejora y realizar las correcciones necesarias y oportunas para las futuras pruebas piloto a realizar.

En el segundo momento de evaluación se contempla integrar, comparar y analizar los resultados de por los menos dos pruebas pilotos más a desarrollarse, las cuales responden a peticiones surgidas a partir de la implementación de esta prueba. Estas futuras pruebas pilotos permitirán robustecer y confrontar los resultados y las experiencias vividas en esta primera implementación.

\section{Resultados}

A continuación, se presentan los resultados más significativos observados en las fases implementadas de acuerdo con el planteamiento esbozado por etapas y definido en la metodología. 


\subsection{Etapa de diagnóstico y diseño}

\subsubsection{Resultados de la evaluación diagnostica}

El conocimiento y uso de redes sociales académicas que manifestaron tener los investigadores del CICAN previo a la celebración de los cursos de formación docente resultó ser limitado, así lo reflejan los datos arrojados en la encuesta aplicada como instrumento de evaluación diagnóstica. En 4 de las 7 redes sociales académicas sobre las que se cuestionó acerca de su conocimiento y uso, los porcentajes combinados correspondientes a los valores "Conozco / No uso", "No conozco / No uso", sobrepasan más del $50 \%$. Los porcentajes relativos al "Uso" solo sobre-pasaron el $50 \%$ en 3 de las 7 redes sociales académicas incluidas en la encuesta (Figura 3).

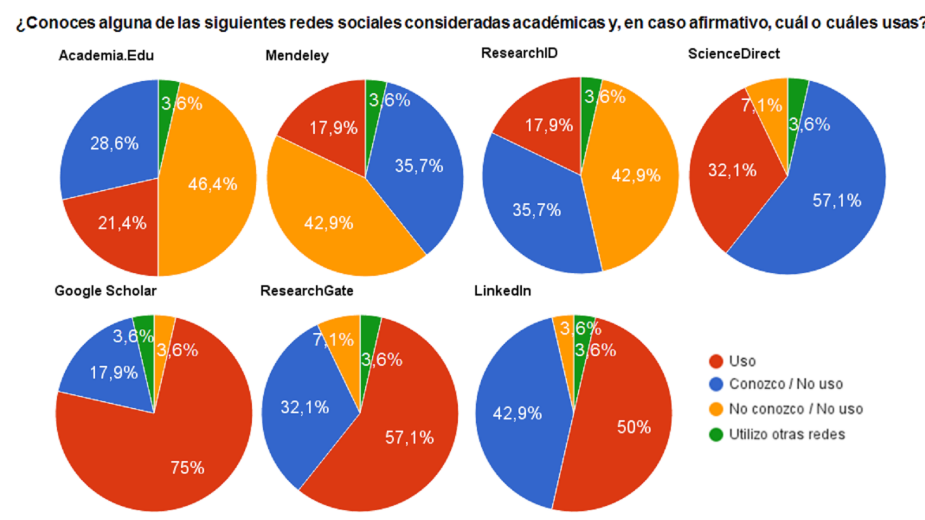

Figura 3. Conocimiento y uso de redes sociales académicas

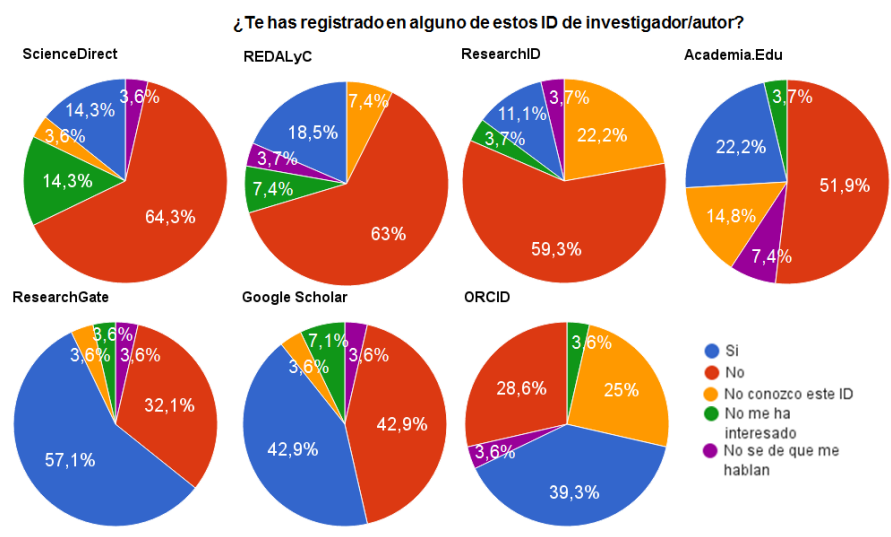

Figura 4. Creación de ID de investigador/autor

Otro indicador que evidenció el limitado conocimiento y uso que tenían y venían haciendo los investigadores del CICAN de las redes sociales académicas, fueron los resultados obtenidos a la pregunta de si contaban con un ID de investigador 0 de autor en alguna de las 7 plataformas que les fueron presentadas en la encuesta. De éstas, solamente una de ellas tuvo un porcentaje mayor al $50 \%$ a la respuesta "Si" (que equivale a haberse registrado), mientras que, en las restantes los porcentajes de "No", "No conozco", "No me ha interesado", "No sé de qué hablan" superaron o fueron cercanos al $50 \%$ (Figuras 4).

Por último, dentro de la evaluación diagnóstica se cuestionó a los participantes sobre las "motivaciones" y las "limitaciones" que afectaban su decisión de usar o no las redes sociales académicas. Entre los resultados más relevantes se encontró que los tres principales motivantes para el uso de las redes sociales académicas entre los investigadores del CICAN están: 1) mantenerse actualizados e informados, 2) difundir sus trabajos hacia el ciudadano común, y 3 ) establecer contactos (Figura 5). Por otro lado, entre los aspectos que los investigadores consideraron que han limitado el uso que ellos hacen de las redes sociales académicas, el factor con mayor mención fue la "falta de tiempo" con un $63 \%$ de referencias, seguido por el "desconocimiento en el uso y manejo" de éstas, con un $37 \%$ (Figura 6 ).

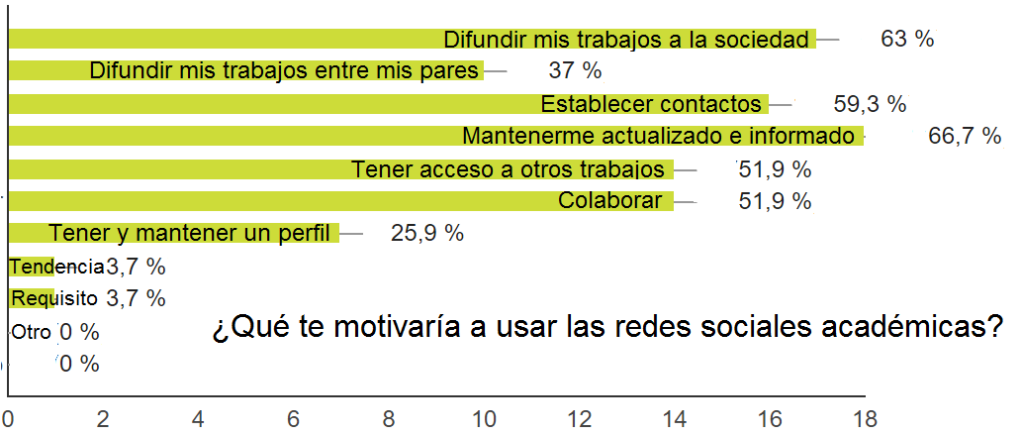

Figura 5. Motivos para el uso de redes sociales consideradas académicas

\begin{tabular}{|c|c|c|c|}
\hline & & \multirow[t]{2}{*}{ No tengo tiempo } & $63 \%$ \\
\hline No domino el ingles & $14,8 \%$ & & \\
\hline \multicolumn{2}{|c|}{ Me resultan dificiles de entender y manejar } & $37 \%$ & \\
\hline Mantener mi privacidad & $18,5 \%$ & & \\
\hline No dispongo de internet & $14.8 \%$ & & \\
\hline \multicolumn{2}{|l|}{ Conexión $\quad 7,4 \%$} & In o han limitado haga & \\
\hline $3,7 \%$ & redes sociales ac & micas? & \\
\hline
\end{tabular}

0

Figura 6. Aspectos que limitan el uso de redes sociales consideradas académicas

Los resultados completos de la evaluación diagnóstica están disponibles para una mejor y libre consulta en el enlace: https://goo.gl/eylnfj. 
5.1.2. Diseño del programa de cursos de formación docente

A partir del análisis de los resultados obtenidos en la evaluación diagnóstica, se detectaron una serie de oportunidades de formación. Con base en estas necesidades, se diseñaron dos cursos de formación docente, los títulos de los cursos fueron: 1) Del curriculum vitae a la identidad digital académica. Herramientas y estrategias para el desarrollo de la visibilidad científica del investigador; y 2) Visibilidad y difusión de los trabajos de investigación. Herramientas y estrategias para aumentar el acceso y el impacto de la producción científica y académica en centros de investigación.

\subsubsection{Diseño de la estrategia}

Esta fase se configuró en tres líneas de acción, las dos primeras de ellas tenían que ver con la creación de perfiles en redes sociales, redes sociales académicas y recursos de la Web 2.0; tanto para cada uno de los profesoresinvestigadores, así como para el propio CICAN. A los profesores-investigadores se le propuso el siguiente conjunto de perfiles a desarrollar voluntariamente: Twitter, G+, Linkedln, SlideSha-re, ResearchGate, Academia.Edu, Google Schoolar, Autores REDALyC, ResearchID, Sco-pus, y Orcid. Cada uno de los anteriores recursos fue abordado en los cursos de formación docente, explicando sus características, uso, beneficios e impactos. Al CICAN y a sus programas de posgrado se le construyeron los siguientes perfiles: Facebook, Twitter, G+, SlideShare. Actualmente, todos los perfiles se encuentran activos y pueden ser consultados y seguidos por cualquier usuario. La tercera de las líneas de la estrategia de implementación se describe a continuación.

\subsubsection{Diseño del entorno tecnológico para la gestión del conocimiento}

Se desarrollaron cuatro herramientas ad-hoc. Tres portales conectados entre sí que permiten gestionar las evidencias generadas tanto en el CICAN, como en sus programas de posgrado. Los tres portales están basados en la versión 7.x de Drupal, un sistema de gestión de contenidos de código abierto: Content Management System (CMS). En segundo lugar, un gestor de blogs basado en WordPress, y otro CMS orientado a la creación de bitácoras en Internet. Aunque los portales mencionados permiten publicar noticias relacionadas con la investigación, los blogs permiten abarcar la vertiente divulgativa de la investigación, así como proporcionarles a estudiantes e investigadores del CICAN un espacio donde compartir conocimientos (http://cican.cusur.udg. $\mathrm{mx} /$ blogs).
5.2. Etapa de desarrollo, implementación y monitoreo

En esta etapa, los resultados que reportamos se derivados de la observación y la reflexión sobre la experiencia que se vivió con la puesta en marcha de la prueba piloto.

\subsubsection{Implementación piloto}

La implementación se vivió en tres momentos. El primero de ellos fue durante la celebración de los cursos de formación docente, éste fue el más visible y el de mayor impacto. Fue en el cara a cara con los profesores-investigadores donde se obtuvo la más detallada radiografía de la percepción y la situación en la que se encontraban los participantes con respecto a la gestión de una identidad digital y al uso y conocimiento de redes sociales académicas y recursos de la Web 2.0 como herramientas de visibilidad académica. El segundo de los momentos, y el menos visible, fue el desarrollo del entorno tecnológico. En esta fase se trabajó de forma interna tanto aspectos tecnológicos como administrativos de la implementación del ecosistema tecnológico. El tercer momento se vivió con la creación y configuración de los perfiles, aquí el trabajo realizado se hizo de manera individual con cada uno de los profesores-investigadores, así como con los directivos del CICAN al momento de generar los perfiles del centro de investigación.

\subsubsection{Monitoreo y análisis de las acciones im- plementadas}

El monitoreo comenzó a realizarse a partir de la conclusión de los cursos de formación docente y con el inicio de la creación y configuración de los perfiles en las redes sociales académicas. Las primeras acciones de monitoreo consistieron en la verificación y retroalimentación de los perfiles creados por los profesores-investigadores, así como en brindar soporte en la resolución de dudas y problemas de configuración. El monitoreo, al igual que el soporte, se mantiene vigente, con la intención de documentar avances, impactos o retrocesos en la gestión de estos perfiles.

\subsection{Etapa de evaluación y mejoras}

\subsubsection{Documentación de la experiencia}

La documentación de la presente experiencia se ha dado a través de diversos mecanismos, esta publicación es uno de ellos, así como también la presentación de este estudio en los trabajos del XXI Encuentros Internacionales sobre Sistemas de Información y Documentación (IBERSID 2016). 
Otros medios de documentación han sido la redacción de una memoria con los contenidos y materiales de los cursos de formación docente para el CICAN, así como para la Red de Bibliotecas de la UdeG (REBIUdeG). Los materiales y la información contenida en la memoria, permitirán replicar y mejorar la implementación de otras pruebas piloto que están por desarrollarse en otros centros de investigación de la Red Universitaria de la UdeG. Estas futuras pruebas piloto han surgido a partir de peticiones realizadas por parte de directivos e investigadores a raíz del conocimiento que tuvieron de la presente implementación.

\subsubsection{Evaluación y correcciones}

Como se mencionó en la metodología, corresponde a este trabajo dar cuenta de los resultados de una primera etapa de evaluación. En esta primera evaluación se aplicaron dos encuestas, la primera recogió la experiencia que vivieron los investigadores en la creación de perfiles académicos; mientras que en la segunda se recogió la opinión y el nivel de satisfacción sobre los contenidos y las actividades de los cursos de formación docente implementados.

Los resultados más significativos se resumen en las respuestas que dieron los participantes al cuestionamiento sobre qué los motivo a dar de alta o actualizar nuevos perfiles. En dichas respuestas se evidencia una reflexión del $90 \%$ de los participantes sobre la importancia de crear y mantener una identidad digital (Figura 7).

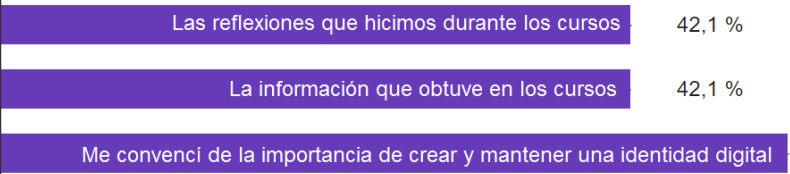

$52,6 \%$

Me senti obligad@ $\quad 10,5 \% \quad$ ¿Qué fue lo que te motivó a dar de alta perfiles nuevos 0 a actualizar tus perfiles existentes?

Figura 7. Motivos para dar de alta nuevos perfiles o actualizar los existentes

Pero en contraste al proceso reflexivo, las respuestas dadas a la pregunta sobre los motivos que todavía les impiden dar de alta nuevos perfiles en redes sociales académicas o actualizar los ya existentes, señalan que aún existe una falta de convencimiento sobre los beneficios del uso de redes sociales académicas y el manejo de una identidad digital (Figura 8).

Con respecto a la satisfacción con los contenidos, los materiales, la estructura y la metodología de los cursos de formación docente, los resultados de la encuesta arrojaron una valoración positiva en todos los rubros. Además, se han recogido comentarios puntuales sobre la necesidad de implementar talleres de formación a mayor detalle en determinadas herramientas $y$ plataformas.

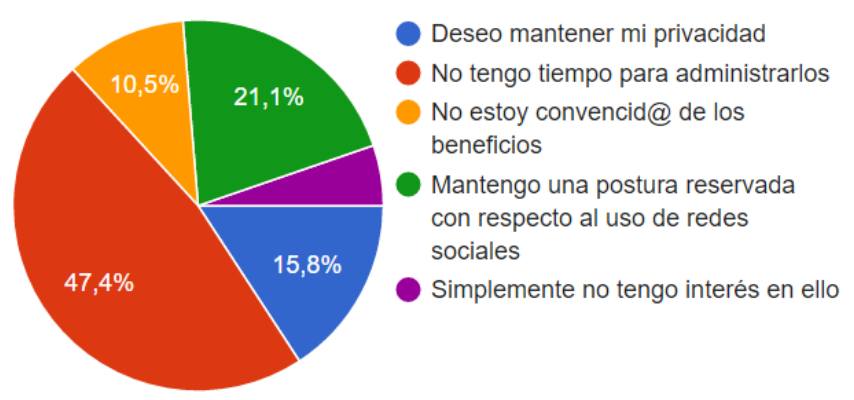

Figura 8. Motivos para no dar de alta perfiles nuevos o actualizar los existentes

Los resultados completos de las evaluaciones realizadas en esta primera fase, están disponibles para su libre consulta en los enlaces: https://goo.gl/oHHBtR y https://goo.gl/m1aZUN.

\section{Conclusiones}

En el ámbito de la docencia y de la investigación, el desarrollo y gestión de una identidad digital ya no es opcional: "es un acto de pura responsabilidad" (Alonso, 2011). Esta responsabilidad, conlleva a la construcción de una reputación y una visibilidad positiva y productiva, que además de visualizar y posicionar el trabajo del investigador, contribuya a la generación, el desarrollo y la visibilidad del conocimiento y la ciencia en su conjunto. Así pues, es necesario que académicos e investigadores adoptemos los entornos y las herramientas digitales como medios y mecanismos de comunicación, transferencia y vinculación de conocimientos e información en nuestro ser y quehacer cotidiano.

Los resultados obtenidos en esta primera prueba piloto, aportan evidencias de la deducción que nos planteamos como hipótesis: en la UdeG existe una escasa y deficiente gestión de identidades digitales entre las comunidades docentes y científicas, así como también de las identidades digitales institucionales de sus centros e institutos de investigación.

Entre las causas que podemos atribuir a partir de la interpretación de los resultados de las encuestas aplicadas, así como de la experiencia vivida en la implementación de la prueba piloto, podemos determinar cuatro causas que condicionan la escasa y deficiente gestión de identidades digitales en la UdeG: 
1) Desconocimiento, desinterés y aislamiento del movimiento académico y científico que se vive en la Web 2.0 por parte de académicos e investigadores de la UdeG.

2) Carencia de programas institucionales de formación y actualización académica y profesional, orientados al desarrollo de habilidades y competencias digitales en el ámbito académico y científico en el marco de la Web 2.0.

3) Obsolescencia y desvinculación de las practicas académicas y de comunicación científica del actual contexto digital y tecnológico en el seno de los centros e institutos de investigación y al interior de la propia universidad.

4) Falta de reconocimientos e incentivos institucionales a prácticas y estrategias de comunicación y difusión del quehacer científico y académico de investigadores y académicos, así como de institutos y centros de investigación.

Consideramos que los factores que condicionan y se expresan en estas cuatro causas, tienen como origen la falta de una estrategia institucional que provea, promueva y reconozca lineamientos, mecanismos y herramientas encaminados al desarrollo y promoción de las identidades digitales de la comunidad académica y científica universitaria, así como también de las instancias e instituciones que conforman a la universidad en su conjunto.

Este primer análisis de los resultados y de la experiencia vivida en la implementación de la prueba piloto, nos permite sugerir que la estrategia planteada, así como el desarrollo de cada una de las etapas y fases propuestas, pueden resultar viables y tomarse en cuenta para la conformación de una hoja de ruta y una guía de buenas prácticas que apoye y soporte la generación de la estrategia a nivel institucional.

\section{Reconocimientos}

Agradecemos la colaboración y las facilidades otorgadas por investigadores y directivos del Centro de Investigaciones en Comportamiento Alimentario y Nutrición (CICAN), así como a las autoridades del Centro Universitario del Sur (CUSur) de la Universidad de Guadalajara (UdeG).

\section{Referencias}

Alonso, J. (2011). Identidad y reputación digital. // Identidad digital y reputación online. Cuadernos de Comunicación evoca. 5 (Julio). http://www.evocaimagen.com/cuader nos/cuadernos5.pdf (2016.09.27).

Alonso Arévalo, J. (2016). Acciones inmediatas para mejorar la visibilidad científica. // Universo Abierto: Blog de la biblioteca de Traducción y Documentación de la Universidad de Salamanca. https://universoabierto.com/ 2016/02/20/acciones-inmediatas-para-mejorar-lavisibilidad-cientifica/ (2016-03-21)

Álvarez de Toledo, M. L. (2013). Cómo difundir y pro- mocionar la identidad digital del profesorado universitario: participar en las redes académicas. Universidad de Oviedo, Biblioteca. Febrero (2013). http:// www.slideshare.net/infobiblio/identidad-digital-para-inves tigadores-redes-sociales-academicas (2016-03-21).

Barrionuevo, L. (2015). Identidad digital y perfil del investigador universitario. Universidad de León Biblioteca Universitaria. http://buleria.unileon.es/handle/ 10612/4878 (2016-03-21).

Borras Gene, O. (2016). Identidad digital: gestión. http://es.slideshare.net/oriolupm/identidad-digitalgestin?next_slideshow=2 (2016-03-21).

Campos Freire, F.; Rúas Araújo, J. (2016). El uso de las redes sociales digitales profesionales y científicas: el caso de las 3 universidades gallegas. // El Profesional de la Información. 25:3 (mayo-junio 2016) 431-440. http://www.elprofesionaldelainformacion.com/contenidos /2016/may/13.pdf (2016-03-21).

Castells, M. (2006). Internet y la sociedad en red. // Contraste: Revista cultural. 43 (2006) 111-113.

Clavel Arroitia, B.; Maruenda Bataller, S. (2011). Uso académico de las redes sociales. // Clavel-Arroitia, B.; Maruenda Bataller, S. (forthcoming). Multiple Voices in Academic and Professional Discourse: Current Issues in Specialised Language Research, Teaching and New Technologies. Newcastle: Cambridge Scholar Publishing. https://www.encuestafacil.com/RespWeb/Cuestiona rios.aspx?EID=652201\&MSJ=NO\#Inicio (2016-03-21).

COPLADI (Coordinación General de Planeación y Desarrollo Institucional) | Universidad de Guadalajara (2016). Numeralia institucional. Guadalajara: Universidad de Guadalajara, 2016. http://copladi.udg.mx/estadistica/ numeralia (2016-03-21).

Cobo, C. y Naval, C. (2013). Digital scholarship: exploration of strategies and Skills for knowledge creation and dissemination. // International Conference on Internet Science Conference Proceedings (pp. 52-59). Brussels: FP7 European Network of Excellence in Internet Science. http://dadun.unav.edu/bitstream/10171/36986/1/Di gital\%20Scholarship.pdf (2016-03-21).

DGEI (Dirección General de Evaluación Institucional) | Universidad Nacional Autónoma de México (2015). Explorador de datos del Estudio Comparativo de Universidades Mexicanas (ExECUM). Ciudad de México: Universidad Nacional Autónoma de México, 2015. http://www.execum.unam.mx/ (2016-03-21).

Esposito, A. (Ed.) (2017). Research 2.0 and the Impact of Digital Technologies on Scholarly Inquiry (pp. 1-343). Hershey, PA: IGI Global. http://www.igi-global.com/book/ research-impact-digital-technologies-scholarly/150396 (2016-03-21).

European Commission (2014). Science 2.0: Science in transition. Public Consultation - Background Document. https://ec.europa.eu/research/consultations/science2.0/background.pdf (2016-03-21).

Fundación Telefónica (2013). Identidad Digital: El nuevo usuario en el mundo digital. Barcelona: Ariel, 2013. http://www.fundaciontelefonica.com/arte_cultura/publica ciones-listado/pagina-item-publicaciones/itempubli/229/ (2016-03-21).

Fernández Vicente, A. (2012). User Profiling. De la autoobjetivación como fuente de control. // Telos. Cuadernos de Comunicación e Innovación. 91 (abril-junio 2012) 6978. https://telos.fundaciontelefonica.com/docs/2012/07/ 26/10390001_4_4_0.pdf\#page=56 (2016-03-21).

Fernández-Marcial, V.; González-Solar LI. (2015). Promoción de la investigación e identidad digital: el caso de la Universidad Da Coruña. // El Profesional de la Información. 24:5 (septiembre-octubre 2015) 656-664. http://www.elprofesionaldelainformacion.com/contenidos 
/2015/sep/14.pdf (2016-03-21).

García Holgado, A.; García Peñalvo, F. J.; Rodríguez Conde, M. J. (2015). Definition of a Technological Ecosystem for Scientific Knowledge Management. // PhD Programme Proceedings of the Third International Conference on Technological Ecosystems for Enhancing Multiculturality (TEEM'15): Porto, Portugal, Oct. 7-9, 2015 (pp. 695-700). New York, NY, USA: ACM, 2015.

Gil, L. (2015a). Uso de las redes sociales en los Centros de Investigación catalanes. Social Media en Investigación. Blog personal de Lydia Gil. http://socialmedia eninvestigacion.com/uso-redes-sociales-centros-deinvestigacion/ (2016-03-21).

Gil, L. (2015b). Uso de las redes sociales en los centros de investigación del CSIC [Infografía]. Social Media en Investigación. Blog personal de Lydia Gil. http://socialmediaeninvestigacion.com/csic-redessociales-generales-cientificas-ii/ (2016-03-21).

Gómez, M.; Roses, S.; Farias, P. (2012). El uso académico de las redes sociales en universitarios. // Comunicar. Revista Científica de Comunicación y Educación. 38 (2012) 131-138. http://www.revistacomunicar.com/in dex. php? contenido $=$ detalles\&numero $=38 \&$ articulo $=38$ 2012-16 (2016-03-21)

González Díaz, C.; Iglesias García, M; Codina, L. (2015) Presencia de las universidades españolas en las redes sociales digitales científicas: caso de los estudios de comunicación. // El Profesional de la In-formación. 24:5 (septiembre-octubre 2015) 640-647. http://www.elprofe sionaldelainformacion.com/contenidos/2015/sep/12.html (2016-03-21)

Laboratorio de Cibermetria | Consejo Superior de Investigaciones Científicas (2015a). Top 2000 Universities by Google Scholar Citations. Madrid: Consejo Superior de Investigaciones Científicas, 2015. http://www.webometrics.info/en/node/169 (2016-03-21).

Laboratorio de Cibermetria | Consejo Superior de Investigaciones Científicas (2015b). Ranking of scientists in Mexico Institutions according to their Google Scholar Citations public profiles. Madrid: Consejo Superior de Investigaciones Científicas, 2015. http://www.webometrics.info/en/node/63 (2016-03-21).

Laboratorio de Cibermetria | Consejo Superior de Investigaciones Científicas (2016). Ranking Mundial de Universidades en la Web. Madrid: Consejo Superior de Investigaciones Científicas, 2016. http://www.webome trics.info/en/world?page=7 (2016-03-21).

Moya Orozco, V. (2015). Más visibilidad para mi investigación. Universidad de Sevilla - Biblioteca. http://www.academia.edu/7181492/M\%C3\%A1s_visibilid ad_para_mi_investigaci\%C3\%B3n (2016-03-21).

Orduña Malea, E.; López Cózar, E. D. (2014). Cómo mejorar la visibilidad de la producción científica de una universidad en la web académica. Grupo de Investigación EC3. Evaluación de la Ciencia y de la Comunicación Científica. http://publicacions.ua.es /Delgado_Orduna.pdf (2016-03-21).

Orduña Malea, E.; López Cózar, E. D. (2016). Creando una identidad académica digital. Grupo de Investigación EC3. Evaluación de la Ciencia y de la Comunicación Científica. https://www.researchgate.net/publication/30 3015378_Creando_una_identidad_academica_digital (2016-06-21).

O'Reilly, Tim (2005). "What Is Web 2.0. Design Patterns and Business Models for the Next Generation of Software". http://www.oreilly.com/pub/a/web2/archive/what-is-web20.html (2016-03-21).

Penkova, S. (2016). Creación y unificación del perfil de investigador. Universidad de Puerto Rico - Biblioteca de
Administración de Empresas. https://www.researchgate. net/publication/292131179_Creacion_y_unificacion_del_ perfil_de_investigador (2016-03-21).

Pérez Subías, M. (2012). Introducción. Identidad Digital. // Telos. Cuadernos de Comunicación e Innovación. 91 (abril-junio 2012) 55-58. https://telos.fundaciontelefonica. com/docs $/ 2012 / 07 / 26 / 10390001$ 4 4 0 0.pdf\#page =56 (2016-03-21)

QS Quacquarelli Symonds (2016). QS World University Rankings. Londres: QS Quacquarelli Symonds, 2016. http://www.topuniversities.com/universities/universidadde-guadalajara-udg\#wur (2016-03-21).

Rathi, D.; Given, L. M. (2010) Research 2.0: A Frame-work for Qualitative and Quantitative Research in Web 2.0 Environments. // Proceedings of the 43rd Hawaii International Conference on System Sciences. https://www.computer.org/csdl/proceedings/hicss/2010/3 869/00/07-07-04.pdf (2016-03-21).

Real Academia Española (RAE) (2104). "Identidad". // Diccionario de la Lengua Española (23a ed.). Madrid, España. http://dle.rae.es/?id=KtmKMfe (2016-03-21).

REBIUN (2011). Ciencia 2.0: aplicación de la web social a la investigación. http://biblioteca.ulpgc.es/files/ciencia 2 0_rebiun_2011.pdf (2016-03-21).

ResearchGate (2015). 8 out of 8 million. Berlin: ResearchGate, 2015. https://www.researchgate.net/blog/ post/8-out-of-8-million (2016-03-21).

ResearchGate (2016). Overview University of Guadalajara. Berlin: ResearchGate, 2016. https://www.researchgate .net/institution/University_of_Guadalajara (2016-03-21).

Rifón Sánchez, A.; Goicoechea Castaño, I.; Fenollera Bolíbar, $\mathrm{M}^{\mathrm{a}}$ I. (2016). Encuesta sobre conocimiento de las redes sociales académicas. http://lyc.survey.webs.uvigo.es/ limesurvey/index.php/145168/lang-es (2016-02-23).

Science Europe (2014). Response to the European Commission Consultation on "Science in Transition". https://goo.gl/z9YZtB (2016-02-23).

SCImago Institutions Rankings (2013). SCImago Institutions Rankings (SIR) Iberoamérica 2013. http://www.scima goir.com/pdf/SIR\%20lber\%202013.pdf (2016-03-21).

SCImago Institutions Rankings (2015). SCImago Institutions Rankings (SIR) Iberoamérica 2015. http://www.scima goir.com/pdf/iber_new/SIR\%20lber\%202015\%20HE.pdf (2016-03-21).

SCImago Institutions Rankings (2016). SCImago Institutions Rankings (SIR) Iberoamérica 2015. http://www.scima goir.com/index.php?sector=Higher\%20educ.\&display $=m$ ap\&year=2008 (2016-03-21).

Universidad de Guadalajara (2015). Informe de Actividades 2015. Guadalajara: Universidad de Guadalajara, 2015. http://www.rectoria.udg.mx/sites/default/files/IA2015mensajeTBP.pdf (2016-03-21).

Uribe Tirado, A. (2015). Percepciones, políticas y formación hacia la visibilidad académica y científica entre profesores-investigadores de la Universidad de Antioquia. BIREDIAL-ISTEC'15. http://eprints.rclis.org/ 28499/1/BIREDIAL.\%20Alejandro\%20Uribe\%20Tirado.p df (2016-03-21).

Zapa-Ros, M. (2011). La investigación y la edición científica en la web social: la ciencia compartida. // RED Docencia Universitaria en la Sociedad del Conocimiento. 3 (2011). http://www.um.es/ead/reddusc/3/zapata_e_ciencia.pdf (2016-03-21).

Enviado: 2016-04-01. Segunda versión: 2016-05-17. Aceptado: 2016-11-13. 